\title{
FUNCTIONAL EQUATIONS SATISFIED BY INTERTWINING OPERATORS OF REDUCTIVE GROUPS
}

\author{
CHEN-BO ZHU
}

\begin{abstract}
This paper generalizes a recent work of Vogan and Wallach [VW] in which they derived a difference equation satisfied by intertwining operators of reductive groups. We show that, associated with each irreducible finitedimensional representation, there is a functional equation relating intertwining operators. In this way, we obtain natural relations between intertwining operators for different series of induced representations.
\end{abstract}

\section{INTRODUCTION}

We use the convention of denoting a Lie group by a capital letter, and denoting its Lie algebra by the corresponding lower case German letter. A subscript $\mathbb{C}$ denotes complexification.

Let $G$ be a real reductive group, $P=M A N$ a parabolic subgroup of $G$ with a given Langlands decomposition [Kn], and $\Phi(P, A)$ the set of positive restricted $A$-roots corresponding to $N$. For $\nu \in \mathfrak{a}_{\mathbb{C}}^{*}$, let $a^{\nu}$ be the character of $A: a^{\nu}=e^{\nu(\log a)}, a \in A$. Let $\sigma$ be an admissible representation of $M$, and $H_{\sigma}$ the representation space. In the sequel, we shall require $\sigma$ to have an infinitesimal character, whose definition is given in (2.1.5). We denote by $I_{P, \sigma, \nu}=\operatorname{Ind}_{M A N}^{G}\left(\sigma \otimes a^{\nu} \otimes 1\right)$ the space of $C^{\infty}$ functions, $f$, from $G$ to $H_{\sigma}$ such that $f(x$ man $)=a^{-(\nu+\rho)} \sigma^{-1}(m) f(x)$, where $m \in M, a \in A, n \in N$, and $\rho=\rho_{P}$, the half sum of the positive restricted $A$-roots counted with multiplicities. $G$ acts on $I_{P, \sigma, \nu}$ by left translation. $I_{P, \sigma, \nu}$ is usually referred to as the generalized principal series.

Let $\bar{P}=M A \bar{N}$ be the opposite parabolic subgroup to $P$. We also define a representation of $G$ by left translation in $I_{\bar{P}, \sigma, \nu}=\operatorname{Ind}_{M A \bar{N}}^{G}\left(\sigma \otimes a^{\nu} \otimes 1\right)$, the space of $C^{\infty}$ functions, $f$, from $G$ to $H_{\sigma}$ such that $f(x m a \bar{n})=$ $a^{-(\nu-\rho)} \sigma^{-1}(m) f(x)$. We recall the standard (but formal) intertwining operator:

$$
J(\bar{P}: P, \sigma, \nu) f(x)=\int_{\bar{N}} f(x \bar{n}) d \bar{n}, \quad f \in I_{P, \sigma, \nu} .
$$

It is well known (see [Kn]) that if $\operatorname{Re}(\nu, \alpha) \geq c=c_{\sigma}$ for $\alpha \in \Phi(P, A)$ and $c_{\sigma}$ some constant depending only on $\sigma$, than the integral defining

Received by the editors January 25, 1991.

1991 Mathematics Subject Classification. Primary 22E30, 22E46; Secondary 15A69.

Key words and phrases. Reductive groups, intertwining operators, functional equations. 
$J(\bar{P}: P, \sigma, \nu)$ converges absolutely. Moreover, in the range of convergence, we have $J(\bar{P}: P, \sigma, \nu): I_{P, \sigma, \nu} \rightarrow I_{\bar{P}, \sigma, \nu}$ and it intertwines the $G$-actions.

The problem of meromorphically continuing the operators $J(\bar{P}: P, \sigma, \nu)$ was resolved in the early seventies by purely analytic methods (see [KS1, KS2]). It was shown, among other things, that in the rank one case, one can in fact analytically continue these operators with respect to the parameter $\nu$, except at negative multiples of some value of $\nu$ where they have simple poles. In this sense, these intertwining operators behave typically like the classical gamma function.

Therefore, it seems tempting and natural to ask whether these intertwining operators indeed have functional equations like the classical gamma function. This was first shown to be so by a recent work of Vogan and Wallach [VW] in which they derived a difference equation satisfied by intertwining operators. The method they employed was by tensoring with a finite-dimensional spherical representation. Clearly, that is the most efficient way of establishing the meromorphic continuation.

The purpose of the present work is to generalize and, at the same time, to explain the Vogan-Wallach result by tensoring with arbitrary finite-dimensional representations. It is fair to say that our main contribution is to show how simple and general the result turns out to be.

Some words about the organization of this paper are in order. In $\S 1(\S \S 1.1-$ 1.2), some very general constructions from multilinear algebra are discussed. These constructions will enable us to define four maps $T, S, U, V$ and obtain most of their properties in a rather transparent way.

In $\S 2$ ( $\S 2.1-2.8)$, two commuting diagrams are proved. The first commuting diagram ( $\$ 2.4)$ relates $T, S$ with intertwining operators. Its proof relies solely on a simple property of some projection operator, which is discussed in $\S 2.2$. Our main result is contained in the second commuting diagram $(\S 2.7)$, which relates $U, V$ with intertwining operators. Its proof uses a critical lemma due to Vogan, the first commuting diagram, and some general properties of $T, S, U, V$. What is important here is that the second commuting diagram gives us a functional equation connecting the intertwining operators $J(\mu, \nu)$ and $J\left(\mu+\mu_{1}, \nu+\nu_{1}\right)$, where $\left(\mu_{1}, \nu_{1}\right)$ is a regular dominant integral infinitesimal character coming from an irreducible finite-dimensional representation of $G$. Moreover, it can be used to express $J(\mu, \nu)$ in terms of $J\left(\mu+\mu_{1}, \nu+\nu_{1}\right)$, not the other way around as in the case of the first commuting diagram. Following [VW], the last section $\S 2.8$ gives some basic properties of the factor $r_{\lambda}^{G}(\Lambda)$, which occurs in the second commuting diagram.

This work is part of the author's Yale thesis. He would like to thank his advisor, Roger Howe, for initiating the project and providing invaluable guidance throughout his graduate study.

\section{Some MUltilineAR ALgebra}

1.1. Generalities on dual and tensor product of vector spaces. Let $F$ be a finitedimensional complex vector space, and $F^{*}$ its complex dual. In this section, all our maps are linear. 
There is a natural map

$$
\operatorname{tr}: F \otimes F^{*} \rightarrow \mathbb{C}
$$

specified by $\operatorname{tr}\left(v \otimes v^{*}\right)=v^{*}(v), v \in F, v^{*} \in F^{*}$. This induces maps

$$
U \otimes F \otimes F^{*} \stackrel{1_{U} \otimes \operatorname{tr}}{\longrightarrow} U
$$

$$
\left(1_{U} \otimes \operatorname{tr}\right)\left(u \otimes v \otimes v^{*}\right)=v^{*}(v) u, \quad u \in U .
$$

Here, $U$ is a complex vector space, possibly infinite dimensional.

We can also think of this as defining contraction maps

$$
\begin{gathered}
\circ v^{*}: U \otimes F \rightarrow U, \\
a \circ v^{*}=\left(1_{U} \otimes \operatorname{tr}\right)\left(a \otimes v^{*}\right), \quad a \in U \otimes F .
\end{gathered}
$$

Thus, $(u \otimes v) \circ v^{*}=v^{*}(v) u, u \in U$.

(1.1.4) Remark. For any dual bases $\left\{y_{i}, y_{i}^{*}\right\}$ of $F$ and $F^{*}$, we have

$$
\sum_{i}\left(a \circ y_{i}^{*}\right) \otimes y_{i}=a, \quad a \in U \otimes F .
$$

(1.1.5) Remark. The contraction mapping has the following property:

$$
\left(1_{U} \otimes A\right)(a) \circ v^{*}=a \circ A^{*}\left(v^{*}\right), \quad a \in U \otimes V, A \in \operatorname{End}(F),
$$

where $A^{*} \in \operatorname{End}\left(F^{*}\right)$ is the adjoint of $A$.

This may be checked by the formula

$$
\begin{aligned}
\left(1_{U} \otimes A\right)(u \otimes v) \circ v^{*} & =(u \otimes A v) \circ v^{*}=v^{*}(A v) u \\
& =\left(A^{*} v^{*}\right)(v) u=(u \otimes v) \circ A^{*}\left(v^{*}\right) .
\end{aligned}
$$

(1.1.6) Remark. Let $F_{1} \subseteq F$ be a subspace. A map $P_{1} \in \operatorname{End}(F)$ is called a projection operator from $F$ onto $F_{1}$ if the image of $P_{1}$ is $F_{1}$ and $\left.P_{1}\right|_{F_{1}}=1_{F_{1}}$. By applying $1_{U} \otimes P_{1}$ to the equation in Remark (1.1.4) and using Remark (1.1.5), we see

$$
\sum_{i}\left(a \circ P_{1}^{*}\left(y_{i}^{*}\right)\right) \otimes P_{1}\left(y_{i}\right)=a, \quad a \in U \otimes F_{1} .
$$

\section{Given a map}

$$
T: X \rightarrow U \otimes F
$$

there is an associated map

$$
T^{\dagger}: X \otimes F^{*} \rightarrow U
$$

defined by the following composition:

$$
X \otimes F^{*} \stackrel{T \otimes 1_{F^{*}}}{\longrightarrow} U \otimes F \otimes F^{*} \stackrel{1_{U} \otimes \operatorname{tr}}{\longrightarrow} U .
$$

Thus, $T^{\dagger}\left(x \otimes v^{*}\right)=T(x) \circ v^{*}$.

We can reconstruct $T$ from $T^{\dagger}$ as follows: Given $S: X \otimes F^{*} \rightarrow U$, we can define $S^{\dagger}: X \rightarrow U \otimes F$ via the diagram:

$$
X \stackrel{j}{\longrightarrow} X \otimes F^{*} \otimes F \stackrel{S \otimes 1_{F}}{\longrightarrow} U \otimes F,
$$


where $j(x)=\sum_{i} x \otimes y_{i}^{*} \otimes y_{i}$, with $\left\{y_{i}\right\}$ any basis of $F$ and $\left\{y_{i}^{*}\right\}$ the dual basis of $F^{*}$. Thus,

$$
S^{\dagger}(x)=\sum_{i} S\left(x \otimes y_{i}^{*}\right) \otimes y_{i} .
$$

(1.1.11) Remark. The expression $\sum_{i} y_{i}^{*} \otimes y_{i} \in F^{*} \otimes F$ is independent of the choice of the dual bases $\left\{y_{i}\right\}$ and $\left\{y_{i}^{*}\right\}$. Under the well-known identification $F^{*} \otimes F \cong \operatorname{End}(F), \sum_{i} y_{i}^{*} \otimes y_{i} \in F^{*} \otimes F$ corresponds to the identity element of $\operatorname{End}(F)$. Here it is worthwhile to point out the following general principle:

For any bilinear map $B(\cdot, \cdot): F^{*} \times F \rightarrow W$, the expression $\sum_{i} B\left(y_{i}^{*}, y_{i}\right) \in$ $W$ is independent of the choice of the dual bases $\left\{y_{i}\right\}$ and $\left\{y_{i}^{*}\right\}$.

Now if we have $T: X \rightarrow U \otimes F$, we then have $T^{\dagger}: X \otimes F^{*} \rightarrow U$ and $\left(T^{\dagger}\right)^{\dagger}: X \rightarrow U \otimes F$

We compute

$$
\left(T^{\dagger}\right)^{\dagger}(x)=\sum_{i} T^{\dagger}\left(x \otimes y_{i}^{*}\right) \otimes y_{i}=\sum_{i}\left(T(x) \circ y_{i}^{*}\right) \otimes y_{i}=T(x)
$$

(by Remark (1.1.4)). Hence, $\left(T^{\dagger}\right)^{\dagger}=T$.

1.2. Generalities on induction, dual, tensor product of representations. Let $G$ be a group and $H$ a subgroup. Let $\rho$ be a finite-dimensional representation of $G$, and $\mu$ a representation of $H$, possibly infinite-dimensional. Then

$$
\rho: G \rightarrow \mathrm{Gl}(F), \quad \mu: H \rightarrow \mathrm{Gl}(U) .
$$

We define $\lambda_{\mu}$ to be the representation of $G$ induced from $\mu$, i.e.,

$$
\lambda_{\mu}=\operatorname{ind}_{H}^{G} \mu=\left\{f: G \rightarrow U \mid f(g h)=\mu^{-1}(h) f(g), \quad g \in G, h \in H\right\} .
$$

$G$ acts on $\lambda_{\mu}$ by left translation.

Since $\rho$ is a finite-dimensional representation, tensoring with $\rho$ is a welldefined, purely algebraic operation.

The following proposition is quite routine, but because it is simple and yet very useful for our later purpose, we include a detailed proof.

(1.2.1) Proposition. The mapping $\alpha: \lambda_{\mu} \otimes \rho \rightarrow \lambda_{\left.\mu \otimes \rho\right|_{H}}$ specified by

$$
\alpha(f \otimes v)(g)=f(g) \otimes \rho(g)^{-1} v, \quad f \in \lambda_{\mu}, v \in F, g \in G,
$$

is a G-isomorphism.

Proof. $\alpha$ is a $G$-map, since

$$
\begin{aligned}
\alpha\left(\left(\lambda_{\mu} \otimes \rho\right)\left(g^{\prime}\right)\left(\sum_{i} f_{i} \otimes v_{i}\right)\right)(g) & =\alpha\left(\sum_{i} \lambda_{\mu}\left(g^{\prime}\right) f_{i} \otimes \rho\left(g^{\prime}\right) v_{i}\right)(g) \\
& =\sum_{i}\left(\lambda_{\mu}\left(g^{\prime}\right) f_{i}\right)(g) \otimes \rho(g)^{-1} \rho\left(g^{\prime}\right) v_{i} \\
& =\sum_{i} f_{i}\left(g^{\prime-1} g\right) \otimes \rho\left(\left(g^{\prime-1} g\right)^{-1} v_{i}\right) \\
& =\alpha\left(\sum_{i} f_{i} \otimes v_{i}\right)\left(g^{\prime-1} g\right)
\end{aligned}
$$


and

$$
\begin{aligned}
\alpha\left(\sum f_{i} \otimes v_{i}\right)(g h) & =\sum_{i} f_{i}(g h) \otimes \rho\left((g h)^{-1}\right) v_{i} \\
& =\sum_{\mu} \mu(h)^{-1} f_{i}(g) \otimes \rho(h)^{-1} \rho\left(g^{-1}\right) v_{i} \\
& =\left(\left.\mu \otimes \rho\right|_{H}\right)(h)^{-1}\left(\sum_{i} f_{i}(g) \otimes \rho(g)^{-1} v_{i}\right) \\
& =\left(\left.\mu \otimes \rho\right|_{H}\right)(h)^{-1}\left(\alpha\left(\sum f_{i} \otimes v_{i}\right)(g)\right) .
\end{aligned}
$$

We construct an inverse of $\alpha$. Let $\phi \in \lambda_{\left.\mu \otimes \rho\right|_{H}}$; then $\phi(g) \in U \otimes F$. Given a basis $\left\{y_{i}\right\}$ of $F$ and a dual basis $\left\{y_{i}^{*}\right\}$ of $F^{*}$, we know that $\left\{\rho(g)^{-1} y_{i}\right\}$ and $\left\{\rho^{*}(g)^{-1} y_{i}^{*}\right\}$ are again dual bases of $F$ and $F^{*}$. Therefore, we can write

$$
\phi(g)=\sum_{i}\left(\phi(g) \circ \rho^{*}(g)^{-1} y_{i}^{*}\right) \otimes \rho(g)^{-1} y_{i} \quad(\text { see Remark (1.1.4)). }
$$

Let

$$
\beta \phi=\sum \phi_{i} \otimes y_{i}
$$

where $\phi_{i}(g)=\phi(g) \circ \rho^{*}(g)^{-1} y_{i}^{*}$.

We check

$$
\begin{gathered}
(\alpha \beta)(\phi)(g)=\alpha\left(\sum \phi_{i} \otimes y_{i}\right)(g)=\sum \phi_{i}(g) \otimes \rho(g)^{-1} y_{i} \\
=\sum_{i}\left(\phi(g) \circ \rho^{*}(g)^{-1} y_{i}^{*}\right) \otimes \rho(g)^{-1} y_{i}=\phi(g) . \\
(\beta \alpha)(f \otimes v)=\sum f_{i} \otimes y_{i},
\end{gathered}
$$

where

$$
\begin{aligned}
f_{i}(g) & =\alpha(f \otimes v)(g) \circ \rho^{*}(g)^{-1} y_{i}^{*}=\left(f(g) \otimes \rho(g)^{-1} v\right) \circ \rho^{*}(g)^{-1} y_{i}^{*} \\
& =f(g) \cdot\left(\rho^{*}(g)^{-1} y_{i}^{*}\right)\left(\rho(g)^{-1} v\right)=f(g) \cdot y_{i}^{*}(v),
\end{aligned}
$$

so

$$
(\beta \alpha)(f \otimes v)=\sum f \cdot y_{i}^{*}(v) \otimes y_{i}=f \otimes v
$$

Now suppose $\nu: H \rightarrow \mathrm{Gl}(X)$ is another representation of $H$, and suppose we have a $H$-intertwining map

$$
\begin{aligned}
T_{0}: X & \rightarrow U \otimes F \\
\nu & \left.\rightarrow \mu \otimes \rho\right|_{H} .
\end{aligned}
$$

This is equivalent to having a $H$-intertwining map

$$
T_{0}^{\dagger}: X \otimes F^{*} \rightarrow U
$$

defined by $T_{0}^{\dagger}\left(x \otimes v^{*}\right)=T_{0}(x) \circ v^{*}$.

The map $T_{0}$ induces a map

$$
\left(T_{0}\right)_{G}: \lambda_{\nu} \rightarrow \lambda_{\left.\mu \otimes \rho\right|_{H}}
$$


by simply composing function values:

$$
\left(T_{0}\right)_{G}(f)(g)=T_{0}(f(g)), \quad f \in \lambda_{\nu}
$$

Composing with $\beta: \lambda_{\left.\mu \otimes \rho\right|_{H}} \rightarrow \lambda_{\mu} \otimes \rho$ gives us a $G$-intertwining map

$$
\beta\left(T_{0}\right)_{G}: \lambda_{\nu} \stackrel{\left(T_{0}\right)_{G}}{\longrightarrow} \lambda_{\left.\mu \otimes \rho\right|_{H}} \stackrel{\beta}{\longrightarrow} \lambda_{\mu} \otimes \rho
$$

which is computed by the formula

$$
\left(\beta\left(T_{0}\right)_{G}\right)(f)=\sum_{i} f_{i} \otimes y_{i}
$$

where $f_{i}(g)=T_{0}(f(g)) \circ \rho^{*}(g)^{-1} y_{i}^{*},\left\{y_{i}\right\}$ is, as above, a basis for $F$, and $\left\{y_{i}^{*}\right\}$ is the dual basis of $F^{*}$.

The map $T_{0}^{\dagger}: X \otimes V^{*} \rightarrow U$ induces a map

$$
\left(T_{0}^{\dagger}\right)_{G}: \lambda_{\left.\nu \otimes \rho^{*}\right|_{H}} \rightarrow \lambda_{\mu}
$$

again by composing function values:

$$
\left(T_{0}^{\dagger}\right)_{G}(\phi)(g)=\left(T_{0}^{\dagger}\right)(\phi(g)), \quad \phi \in \lambda_{\left.\nu \otimes \rho^{*}\right|_{H}} .
$$

Composing with $\alpha: \lambda_{\nu} \otimes \rho^{*} \rightarrow \lambda_{\nu \otimes \rho^{*} \mid H}$ gives us a $G$-intertwining map

$$
\left(T_{0}^{\dagger}\right)_{G} \alpha: \lambda_{\nu} \otimes \rho^{*} \stackrel{\alpha}{\longrightarrow} \lambda_{\nu \otimes \rho^{*} \mid H} \stackrel{\left(T_{0}^{\dagger}\right)_{G}}{\longrightarrow} \lambda_{\mu},
$$

which is computed by the formula

$$
\left(\left(T_{0}^{\dagger}\right)_{G} \alpha\right)\left(f \otimes v^{*}\right)(g)=T_{0}(f(g)) \circ \rho^{*}(g)^{-1} v^{*}, \quad f \in \lambda_{\nu} .
$$

Let us compare (1.2.6) with (1.2.9). If $f \in \lambda_{\nu}$, and $v^{*} \in F^{*}$, then we compute

$$
\left(\beta\left(T_{0}\right)_{G}\right)^{\dagger}\left(f \otimes v^{*}\right)=\left(\beta\left(T_{0}\right)_{G}\right) f \circ v^{*}=\left(\sum f_{i} \otimes y_{i}\right) \circ v^{*}=\sum f_{i} \cdot v^{*}\left(y_{i}\right),
$$

where

$$
f_{i}(g)=T_{0}(f(g)) \circ \rho^{*}(g)^{-1} y_{i}^{*}
$$

Therefore,

$$
\begin{aligned}
\left(\left(\beta\left(T_{0}\right)_{G}\right)^{\dagger}\right)\left(f \otimes v^{*}\right)(g) & =T_{0}(f(g)) \circ \sum v^{*}\left(y_{i}\right) \rho^{*}(g)^{-1} y_{i}^{*} \\
& =T_{0}(f(g)) \circ \rho^{*}(g)^{-1} v^{*} .
\end{aligned}
$$

In other words,

$$
\left(\beta\left(T_{0}\right)_{G}\right)^{\dagger}=\left(T_{0}^{\dagger}\right)_{G} \alpha
$$

Suppose we have a submodule

$$
\left.\rho_{1} \subseteq \rho\right|_{H}, \quad F_{1} \subseteq F .
$$

Let $P_{1}$ be a projection map from $F$ onto $F_{1}$ (not necessarily a $H$-map) (see Remark (1.1.6) for the definition of such a map). Suppose the image of $T_{0} \subseteq U \otimes F_{1}$. Then

$$
T_{0}=\left(1_{U} \otimes P_{1}\right) T_{0}
$$


Hence if $f \in \lambda_{\nu}$ and $v^{*} \in F^{*}$, we can compute

$$
\begin{aligned}
& \left(\beta\left(T_{0}\right)_{G}\right)^{\dagger}\left(f \otimes v^{*}\right)(g)=\left(T_{0}^{\dagger}\right)_{G} \alpha\left(f \otimes v^{*}\right)(g) \\
& \quad=T_{0}(f(g)) \circ \rho^{*}(g)^{-1} v^{*} \\
& \quad=\left(1_{U} \otimes P_{1}\right) T_{0}(f(g)) \circ \rho^{*}(g)^{-1} v^{*} \\
& \quad=T_{0}(f(g)) \circ P_{1}^{*}\left(\rho^{*}(g)^{-1} v^{*}\right) \quad \text { by Remark (1.1.5)). }
\end{aligned}
$$

Therefore, we have

$$
\left(\beta\left(T_{0}\right)_{G}\right)(f)=\sum f_{i} \otimes y_{i}, \quad f \in \lambda_{\nu},
$$

where $f_{i}=\left(\beta\left(T_{0}\right)_{G}\right)^{\dagger}\left(f \otimes y_{i}^{*}\right)=T_{0}(f(g)) \circ P_{1}^{*}\left(\rho^{*}(g)^{-1} y_{i}^{*}\right)$.

\section{NATURAL RELATIONS BETWEEN INTERTWINING OPERATOR}

2.1. Preliminaries. Let $\mathfrak{g}$ be a complex reductive Lie algebra. Choose a Borel subalgebra $\mathfrak{b}$ of $\mathfrak{g}$, and thus a Cartan subalgebra $\mathfrak{h} \subseteq \mathfrak{b}$.

(2.1.1) The irreducible finite-dimensional representations of $\mathfrak{g}$ can be parametrized by their highest weights with respect to $\mathfrak{b}$, which are dominant integral (see $[\mathrm{Hu}])$.

(2.1.2) Let $\mathscr{U}(\mathfrak{g})$ be the universal enveloping algebra of $\mathfrak{g}$, and $\mathscr{Z}(\mathfrak{g})$ its center. We know from Harish-Chandra (see [V1], for example) that the homomorphisms of $\mathscr{Z}(\mathfrak{g})$ into $\mathbb{C}$ can be parametrized by elements of $\mathfrak{h}^{*} / W_{G}$, the set of Weyl group orbits in $\mathfrak{h}^{*}$. Let $C_{G}$ be the positive Weyl chamber specified by our choice of $\mathfrak{b}$. It is a fundamental domain for the action of $W_{G}$ on $\mathfrak{h}^{*}$. For $\lambda \in C_{G} \subseteq \mathfrak{h}^{*}$, the corresponding homomorphism of $\mathscr{Z}(\mathfrak{g})$ into $\mathbb{C}$ is denoted by $\chi_{\lambda}$.

We fix our parabolic subgroup $P=M A N$ as in the Introduction.

Choose a Borel subalgebra $\mathfrak{b}_{0}$ of $\mathfrak{m}_{\mathbb{C}}$, and thus a Cartan subalgebra $\mathfrak{h}_{0} \subseteq \mathfrak{b}_{0}$. Then $\mathfrak{b}=\mathfrak{b}_{0} \oplus \mathfrak{n}_{\mathbb{C}}$ is a Borel subalgebra of $\mathfrak{g}_{\mathbb{C}}$, and $\mathfrak{h}=\mathfrak{h}_{0} \oplus \mathfrak{a}_{\mathbb{C}}$ is a Cartan subalgebra contained in $\mathfrak{b}$.

(2.1.3) We can apply (2.1.1) to the Lie algebras $\mathfrak{m}_{\mathbb{C}}$ and $\mathfrak{g}_{\mathbb{C}}$. Thus if $\mu_{1} \in \mathfrak{h}_{0}^{*}$ is dominant integral with respect to $\mathfrak{b}_{0}$ and in the weight lattice, the unique irreducible finite-dimensional representation of $M$ with this highest weight is denoted by $\sigma_{\mu_{1}}$. Similarly if $\left(\mu_{1}, \nu_{1}\right) \in \mathfrak{h}^{*}$ is dominant integral with respect to $\mathfrak{b}, \mu_{1} \in \mathfrak{h}_{0}^{*}, \nu_{1} \in \mathfrak{a}_{\mathbb{C}}^{*}$, the corresponding irreducible finite-dimensional representation of $G$ is denoted by $F_{\mu_{1}, \nu_{1}}$.

For a finite-dimensional representation $(\pi, F)$ of $G$, denote by

$$
F^{N}=\{v \in F \mid \pi(n) v=v \quad \forall n \in N\}
$$

the space of $N$-fixed vectors. Similarly $F^{\bar{N}}$ denotes the space of $\bar{N}$-fixed vectors.

We have the following standard lemma [W1].

(2.1.4) Lemma. Let $(\pi, F)$ be an irreducible finite-dimensional representation of $G$, and $\left(\pi^{*}, F^{*}\right)$ the contragradient representation. Then $F^{N}$ and $\left(F^{*}\right)^{\bar{N}}$ 
are irreducible representations of $M A$. Moreover, as representations of $M A$, they are contragradient to each other.

Thus in the notation of this section,

$$
F_{\mu_{1}, \nu_{1}}^{N} \cong \sigma_{\mu_{1}} \otimes a^{\nu_{1}}
$$

as $M A$-modules.

(2.1.5) Given an admissible representation $(\pi, V)$ of $G$, there is a resulting representation of $\mathscr{U}\left(\mathrm{g}_{\mathbb{C}}\right)$ on $V_{0}$, the space of $K$-finite vectors, where $K$ is a maximal compact subgroup of $G$. If $\mathscr{Z}\left(\mathfrak{g}_{\mathbb{C}}\right)$ acts by scalars on $V_{0}$, the corresponding homomorphism

$$
\chi: \mathscr{Z}\left(\mathfrak{g}_{\mathbb{C}}\right) \rightarrow \mathbb{C},
$$

defined by

$$
z \cdot x=\chi(z) x, \quad x \in V_{0}, \quad z \in \mathscr{Z}\left(\mathfrak{g}_{\mathbb{C}}\right),
$$

is called the infinitesimal character of $\pi$.

More generally, if for some integer $d>0$,

$$
(z-\chi(z))^{d} x=0 \text { for all } x \in V_{0}, \quad z \in \mathscr{Z}\left(\mathfrak{g}_{\mathbb{C}}\right),
$$

we say $\pi$ has the generalized infinitesimal character $\chi$.

If $\chi=\chi_{\lambda}, \lambda \in C_{G}$ (see (2.1.2)), we shall also say $\pi$ has the infinitesimal character $\lambda$ (resp. generalized infinitesimal character $\lambda$ ).

We quote the following result from $[\mathrm{Kn}]$. Let $(\pi, V)$ be a Harish-Chandra module, i.e., a finitely generated admissible $G$-module. Then there exist linear functionals $\lambda_{1}, \ldots, \lambda_{l}$ on $\mathfrak{h}, \mathscr{U}\left(\mathfrak{g}_{\mathbb{C}}\right)$-invariant subspaces $V_{1}, \ldots, V_{l}$ of $V_{0}$, and an integer $d>0$ such that

(a) $\lambda_{1}, \ldots, \lambda_{l}$ are mutually inequivalent under the Weyl group.

(b) $V_{0}=V_{1} \oplus \cdots \oplus V_{l}$.

(c) $\left(z-\chi_{\lambda_{j}}(z)\right)^{d}$ acts as the zero operator in $V_{j}$ for all $z \in \mathscr{Z}\left(\mathfrak{g}_{\mathbb{C}}\right)$.

Thus, we have a canonically defined projection operator, denoted by $P_{\lambda}^{G}$, from the space of $K$-finite vectors of $V$ to the subspace with the generalized infinitesimal character $\lambda$.

Similarly, if $E$ is a Harish-Chandra module of $M$, we denote by $P_{\mu}^{M}$ the projection operator from the space of $K \cap M$ finite vectors of $E$ to the subspace with the generalized infinitesimal character $\mu \in C_{M}$, the positive Weyl chamber specified by our choice of $\mathfrak{b}_{0}$ in $\mathfrak{m}_{\mathbb{C}}$.

2.2. Two projection maps: $P_{N}$ and $P_{\bar{N}}$. From now on, we fix $F=F_{\mu_{1}, \nu_{1}}$, an irreducible finite-dimensional representation of $G$.

(2.2.1) Lemma. We have the following direct sum decompositions:

$$
\begin{aligned}
& F=F^{N} \oplus \overline{\mathfrak{n}} F, \\
& F^{*}=\left(F^{*}\right)^{\bar{N}} \oplus \mathfrak{n} F^{*} \quad \text { (as MA-modules), }
\end{aligned}
$$

where $\mathfrak{n}$ and $\overline{\mathfrak{n}}$ are Lie algebras of $N$ and $\bar{N}$, respectively.

Proof. Use $\mathfrak{g}_{\mathbb{C}}=\overline{\mathfrak{n}}+\mathfrak{m}+\mathfrak{a}+\mathfrak{n}$, the Poincare-Birkhoff-Witt theorem, and the irreducibility of $F$. We leave the details to the reader. 
We denote by $P_{N}$ and $P_{\bar{N}}$, the projections to $F^{N}$ and $\left(F^{*}\right)^{\bar{N}}$, according to the decompositions $(2.2 .2)$ and (2.2.3), respectively. They are $M A$ homomorphisms.

We prove the following properties of $P_{N}$ and $P_{\bar{N}}$.

(2.2.4) Proposition. (i) $P_{N}(\bar{n} v)=P_{N}(v), \bar{n} \in \bar{N}, v \in F ; P_{\bar{N}}\left(n v^{*}\right)=P_{\bar{N}}\left(v^{*}\right)$, $n \in N, v^{*} \in F^{*}$.

(ii) $P_{N}$ and $P_{\bar{N}}$ are adjoint to each other as elements of $\operatorname{End}(F)$ and $\operatorname{End}\left(F^{*}\right)$. Proof. (i) By definition, we have $P_{N}(X v)=0, X \in \overline{\mathfrak{n}}, v \in F$. From the well-known relationship between a Lie group and its Lie algebra, it follows that $P_{N}(\bar{n} v)=P_{N}(v), \quad \bar{n} \in \bar{N}, v \in F$.

(ii) Let

$$
\begin{aligned}
& v=v_{1}+v_{2}, \quad v_{1} \in F^{N}, v_{2} \in \overline{\mathfrak{n}} F, \\
& v^{*}=v_{1}^{*}+v_{2}^{*}, \quad v_{1}^{*} \in\left(F^{*}\right)^{\bar{N}}, v_{2}^{*} \in \mathfrak{n} F^{*} .
\end{aligned}
$$

Since $v_{1}^{*} \in\left(F^{*}\right)^{\bar{N}}$, we have $v_{1}^{*}(X v)=-\left(X v_{1}^{*}\right)(v)=0$ for $X \in \overline{\mathrm{n}}, v \in F$. Therefore $v_{1}^{*}\left(v_{2}\right)=0$, and $v_{2}^{*}\left(v_{1}\right)=0$ by a similar computation. Hence,

$$
\begin{aligned}
& \left(P_{\bar{N}} v^{*}\right)(v)=v_{1}^{*}(v)=v_{1}^{*}\left(v_{1}\right), \\
& v^{*}\left(P_{N}(v)\right)=v^{*}\left(v_{1}\right)=v_{1}^{*}\left(v_{1}\right) .
\end{aligned}
$$

2.3. Definitions of four maps $T, S, U, V$. Let $F=F_{\mu_{1}, \nu_{1}}$ be the irreducible representation of $G$ such that $F^{N}=\sigma_{\mu_{1}} \otimes a^{\nu_{1}}$ as representations of $M A$. Let $\sigma$ be an admissible representation of $M$ with an infinitesimal character $\mu \in C_{M}$ (see $\S 2.1$ ). We shall write $\sigma_{\mu}$ instead of $\sigma$ for the sake of notation.

Consider $I_{P, \mu, \nu}=\operatorname{Ind}_{M A N}^{G}\left(\sigma_{\mu} \otimes a^{\nu} \otimes 1\right)$. Let

$$
\sigma_{\mu+\mu_{1}} \stackrel{\text { def }}{=} P_{\mu+\mu_{1}}^{M}\left(\sigma_{\mu} \otimes \sigma_{\mu_{1}}\right) \text {. }
$$

We recall here that $P_{\mu+\mu_{1}}^{M}$ is the projection operator onto the generalized infinitesimal character $\mu+\mu_{1}$. We caution the reader that $\sigma_{\mu_{1}}$ has the infinitesimal character $\mu_{1}+\rho_{M}$ instead of $\mu_{1}$, where $\rho_{M}$ is the half sum of the positive $\mathfrak{h}_{0}$ roots in $\mathfrak{m}_{\mathbb{C}}$.

Let

$$
I_{P, \mu+\mu_{1}, \nu+\nu_{1}} \stackrel{\text { def }}{=} \operatorname{Ind}_{M A N}^{G}\left(\sigma_{\mu+\mu_{1}} \otimes a^{\nu+\nu_{1}} \otimes 1\right) .
$$

We shall define below four maps $T, S, U, V$, which fit into the following two diagrams:

$$
\begin{aligned}
& I_{P, \mu, \nu} \otimes F_{\mu_{1}, \nu_{1}} \stackrel{J(\bar{P}: P, \mu, \nu) \otimes I}{\longrightarrow} I_{\bar{P}, \mu, \nu} \otimes F_{\mu_{1}, \nu_{1}} \\
& \uparrow T \\
& I_{P, \mu+\mu_{1}, \nu+\nu_{1}} \stackrel{J\left(\bar{P}: P, \mu+\mu_{1}, \nu+\nu_{1}\right)}{\longrightarrow} I_{\bar{P}, \mu+\mu_{1}, \nu+\nu_{1}} \\
& I_{P, \mu+\mu_{1}, \nu+\nu_{1}} \otimes F_{\mu_{1}, \nu_{1}}^{*} \stackrel{J\left(\bar{P}: P, \mu+\mu_{1}, \nu+\nu_{1}\right) \otimes I}{\longrightarrow} I_{\bar{P}, \mu+\mu_{1}, \nu+\nu_{1}} \otimes F_{\mu_{1}, \nu_{1}}^{*}
\end{aligned}
$$

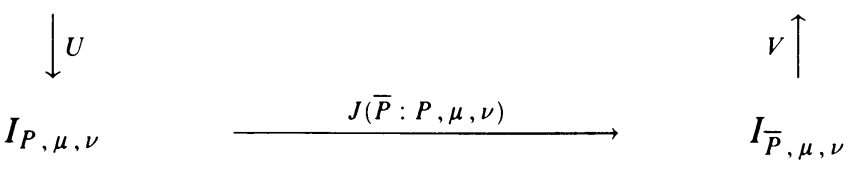


The reader is referred to $\S \S 1.1$ and 1.2 for various notation used in the following.

We have the natural inclusion:

$$
\sigma_{\mu+\mu_{1}} \hookrightarrow \sigma_{\mu} \otimes \sigma_{\mu_{1}} .
$$

This will induce a natural inclusion of $\sigma_{\mu+\mu_{1}} \otimes a^{\nu+\nu_{1}} \otimes 1$ into

$$
\begin{aligned}
\left(\sigma_{\mu} \otimes \sigma_{\mu_{1}}\right) \otimes a^{\nu+\nu_{1}} \otimes 1 & =\left(\sigma_{\mu} \otimes a^{\nu} \otimes 1\right) \otimes\left(\sigma_{\mu_{1}} \otimes a^{\nu_{1}} \otimes 1\right) \\
& =\left(\sigma_{\mu} \otimes a^{\nu} \otimes 1\right) \otimes\left(F_{\mu_{1}, \nu_{1}}\right)^{N},
\end{aligned}
$$

but $\left(F_{\mu_{1}, \nu_{1}}\right)^{N}$ is a $M A$-submodule of $\left.\left(F_{\mu_{1}, \nu_{1}}\right)\right|_{P}$, so

$$
T_{0}:\left.\sigma_{\mu+\mu_{1}} \otimes a^{\nu+\nu_{1}} \otimes 1 \hookrightarrow\left(\sigma_{\mu} \otimes a^{\nu} \otimes 1\right) \otimes\left(F_{\mu_{1}, \nu_{1}}\right)\right|_{P}
$$

as $P$-modules.

Therefore, we have a natural G-map

$$
\begin{aligned}
T=\beta\left(T_{0}\right)_{G}: & \operatorname{Ind}_{P}^{G}\left(\sigma_{\mu+\mu_{1}} \otimes a^{\nu+\nu_{1}} \otimes 1\right) \\
& \stackrel{\left(T_{0}\right)_{G}}{\longrightarrow} \operatorname{Ind}_{P}^{G}\left(\left.\left(\sigma_{\mu} \otimes a^{\nu} \otimes 1\right) \otimes\left(F_{\mu_{1}, \nu_{1}}\right)\right|_{P}\right) \\
& \stackrel{\beta}{\cong} \operatorname{Ind}_{P}^{G}\left(\sigma_{\mu} \otimes a^{\nu} \otimes 1\right) \otimes F_{\mu_{1}, \nu_{1}} .
\end{aligned}
$$

Since the image of $T_{0} \subseteq\left(\sigma_{\mu} \otimes a^{\nu} \otimes 1\right) \otimes\left(F_{\mu_{1}, \nu_{1}}\right)^{N}$, the above map $T$ can be computed as follows:

$$
T(f)=\sum_{i} f_{i} \otimes y_{i}, \quad f \in I_{P, \mu+\mu_{1}, \nu+\nu_{1}}
$$

where $f_{i}(g)=f(g) \circ P_{\bar{N}}\left(g^{-1} y_{i}^{*}\right)$, with $\left\{y_{i}\right\}$ and $\left\{y_{i}^{*}\right\}$ dual bases of $F$ and $F^{*}$ (see (1.2.14) and Proposition (2.2.4)).

Let $U$ be the following natural $G$-map:

$$
\begin{aligned}
U=\left(T_{0}^{\dagger}\right)_{G} \alpha: & \operatorname{Ind}_{P}^{G}\left(\sigma_{\mu+\mu_{1}} \otimes a^{\nu+\nu_{1}} \otimes 1\right) \otimes F_{\mu+\mu_{1}}^{*} \\
& \stackrel{\alpha}{\cong} \operatorname{Ind}_{P}^{G}\left(\left(\sigma_{\mu+\mu_{1}} \otimes a^{\nu+\nu_{1}} \otimes 1\right) \otimes F_{\mu_{1}, \nu_{1}}^{*}\right) \\
& \stackrel{\left(T_{0}^{\dagger}\right)_{G}}{\longrightarrow} \operatorname{Ind}_{P}^{G}\left(\sigma_{\mu} \otimes a^{\nu} \otimes 1\right) .
\end{aligned}
$$

We know by (1.2.11) that $U=T^{\dagger}$, i.e.,

$$
U\left(f \otimes v^{*}\right)=T(f) \circ v^{*} .
$$

By (1.2.13), we have

$$
U\left(f \otimes v^{*}\right)(g)=f(g) \circ P_{\bar{N}}\left(g^{-1} v^{*}\right), \quad f \in I_{P, \mu+\mu_{1}, \nu+\nu_{1}} .
$$

Also, we have the following natural epimorphism:

$$
\begin{aligned}
& \sigma_{\mu} \otimes \sigma_{\mu_{1}} \rightarrow \sigma_{\mu+\mu_{1}}, \\
& u \otimes u_{1} \rightarrow P_{\mu+\mu_{1}}^{M}\left(u \otimes u_{1}\right), \quad u \in \sigma_{\mu}, u_{1} \in \sigma_{\mu_{1}} .
\end{aligned}
$$

This will induce a $M A$-map

$$
\sigma_{\mu} \otimes a^{\nu} \otimes\left(F_{\mu_{1}, \nu_{1}}\right)^{N} \rightarrow \sigma_{\mu+\mu_{1}} \otimes a^{\nu+\nu_{1}},
$$

where $\left(F_{\mu_{1}, \nu_{1}}\right)^{N}$ is a $M A$-quotient of $F_{\mu_{1}, \nu_{1}}$ under the map

$$
P_{N}: F_{\mu_{1}, \nu_{1}} \rightarrow\left(F_{\mu_{1}, \nu_{1}}\right)^{N} .
$$


So we have

$$
\sigma_{\mu} \otimes a^{\nu} \otimes F_{\mu_{1}, \nu_{1}} \rightarrow \sigma_{\mu} \otimes a^{\nu} \otimes\left(F_{\mu_{1}, \nu_{1}}\right)^{N} \rightarrow \sigma_{\mu+\mu_{1}} \otimes a^{\nu+\nu_{1}}
$$

Since the map $P_{N}$ has the property $P_{N}(\bar{n} v)=P_{N}(v)$ for $\bar{n} \in \bar{N}$ (Proposition (2.2.4)), the composition of the above two epimorphisms yields an epimorphism

$$
S_{0}:\left.\left(\sigma_{\mu} \otimes a^{\nu} \otimes 1\right) \otimes F_{\mu_{1}, \nu_{1}}\right|_{\bar{P}} \rightarrow \sigma_{\mu+\mu_{1}} \otimes a^{\nu+\nu_{1}} \otimes 1
$$

as $\bar{P}=M A \bar{N}$-modules.

Therefore, we have a natural $G$-map

$$
\begin{aligned}
S=\left(S_{0}\right)_{G} \alpha: & \operatorname{Ind} \frac{G}{P}\left(\sigma_{\mu} \otimes a^{\nu} \otimes 1\right) \otimes F_{\mu_{1}, \nu_{1}} \\
& \stackrel{\alpha}{\cong} \operatorname{Ind} \frac{G}{P}\left(\left(\sigma_{\mu} \otimes a^{\nu} \otimes 1\right) \otimes F_{\mu_{1}, \nu_{1}} \mid \bar{P}\right) \\
& \stackrel{\left(S_{0}\right)_{G}}{\rightarrow} \operatorname{Ind} \frac{G}{P}\left(\sigma_{\mu+\mu_{1}} \otimes a_{\nu+\nu_{1}} \otimes 1\right) .
\end{aligned}
$$

It can be computed as follows:

$$
S(h \otimes v)(g)=P_{\mu+\mu_{1}}^{M}\left(h(g) \otimes P_{N}\left(g^{-1} v\right)\right), \quad h \in I_{\bar{P}, \mu, \nu} .
$$

Let $V$ be the following natural $G$-map,

$$
\begin{aligned}
V=\beta\left(S_{0}^{\dagger}\right)_{G}: & \operatorname{Ind} \frac{G}{P}\left(\sigma_{\mu} \otimes a^{\nu} \otimes 1\right) \\
& \stackrel{\left(S_{0}^{\dagger}\right)_{G}}{\longrightarrow} \operatorname{Ind} \frac{G}{P}\left(\sigma_{\mu+\mu_{1}} \otimes a^{\nu+\nu_{1}} \otimes 1 \otimes F_{\mu_{1}, \nu_{1}}^{*} \mid \bar{P}\right) \\
& \stackrel{\beta}{\cong} \operatorname{Ind} \frac{G}{P}\left(\sigma_{\mu+\mu_{1}} \otimes a^{\nu+\nu_{1}} \otimes 1\right) \otimes F_{\mu_{1}, \nu_{1}}^{*} .
\end{aligned}
$$

By (1.2.11), $S=V^{\dagger}$. Therefore,

$$
V(h)=\sum S\left(h \otimes y_{i}\right) \otimes y_{i}^{*},
$$

or explicitly, by $(2.3 .11)$

$$
V(h)=\sum h_{i} \otimes y_{i}^{*}, \quad h \in I_{\bar{P}, \mu, \nu},
$$

where $h_{i}(g)=P_{\mu+\mu_{1}}^{M}\left(h(g) \otimes P_{N}\left(g^{-1} y_{i}\right)\right)$.

(2.3.15) Remark. When $\mu_{1}=0$, i.e., $M$ acts trivially on $F^{N}$, by a theorem of Helgason [He], $F^{N}$ is one dimensional and $F$ is spherical in the sense that it has a $K$-fixed vector, where $K$ is a maximal compact subgroup of $G$, as before. The above definitions of $T, S, U, V$ reduce to the formulas of $T$, $S, U, V$ given in [VW].

2.4. First commuting diagram. Choose a constant $c$ such that, if $\operatorname{Re}(\nu, \alpha) \geqslant c$ for $\alpha \in \Phi(P, A)$, then both integrals defining $J(\bar{P}: P, \mu, \nu)$ and $I(\bar{P}$ : $\left.P, \mu+\mu_{1}, \nu+\nu_{1}\right)$ converge absolutely (see the Introduction).

(2.4.1) Proposition. If $\operatorname{Re}(\nu, \alpha) \geqslant c$ for $\alpha \in \Phi(P, A)$, then the following diagram commutes:

$$
\begin{array}{ccc}
I_{P, \mu, \nu} \otimes F_{\mu_{1}, \nu_{1}} & \stackrel{J(\bar{P}: P, \mu, \nu) \otimes I}{\longrightarrow} & I_{\bar{P}, \mu, \nu} \otimes F_{\mu_{1}, \nu_{1}} \\
\uparrow T & & S \downarrow \\
I_{P, \mu+\mu_{1}, \nu+\nu_{1}} \stackrel{J\left(\bar{P}: P, \mu+\mu_{1}, \nu+\nu_{1}\right)}{\longrightarrow} & I_{\bar{P}, \mu+\mu_{1}, \nu+\nu_{1}}
\end{array}
$$


Proof. Let $\left\{y_{i}\right\}$ and $\left\{y_{i}^{*}\right\}$ be dual bases of $F_{\mu_{1}, \nu_{1}}$ and $F_{\mu_{1}, \nu_{1}}^{*}$, as before. Set $f_{i}(g)=f(g) \circ P_{\bar{N}}\left(g^{-1} y_{i}^{*}\right)$ for $f \in I_{P, \mu+\mu_{1}, \nu+\nu_{1}}$. Then, by (2.3.5),

$$
T(f)=\sum_{i} f_{i} \otimes y_{i}
$$

Thus,

$$
\begin{aligned}
S(J(\bar{P}: P, \mu, \nu) \otimes I) T f & =\sum_{i} S(J(\bar{P}: P, \mu, \nu) \otimes I)\left(f_{i} \otimes y_{i}\right) \\
& =\sum_{i} S\left(J(\bar{P}: P, \mu, \nu) f_{i} \otimes y_{i}\right) .
\end{aligned}
$$

By the formula of $S$ in (2.3.11), we have

$$
\begin{aligned}
S(J & \left.(\bar{P}: P, \mu, \nu) f_{i} \otimes y_{i}\right)(g) \\
& =P_{\mu+\mu_{1}}^{M}\left(\left(J(\bar{P}: P, \mu, \nu) f_{i}\right)(g) \otimes P_{N}\left(g^{-1} y_{i}\right)\right) \\
& =P_{\mu+\mu_{1}}^{M}\left(\int_{\bar{N}} f_{i}(g \bar{n}) d \bar{n} \otimes P_{N}\left(g^{-1} y_{i}\right)\right) \\
& =P_{\mu+\mu_{1}}^{M}\left(\int_{\bar{N}}\left(f(g \bar{n}) \circ P_{\bar{N}}\left((g \bar{n})^{-1} y_{i}^{*}\right)\right) \otimes P_{N}\left(g^{-1} y_{i}\right) d \bar{n}\right) .
\end{aligned}
$$

Since

$$
P_{N}\left(g^{-1} y_{i}\right)=P_{N}\left(\bar{n}^{-1} g^{-1} y_{i}\right)=P_{N}\left((g \bar{n})^{-1} y_{i}\right), \quad \text { (by Proposition (2.2.4)), }
$$

the expression inside the integral (2.4.3) is equal to

$$
\left(f(g \bar{n}) \circ P_{\bar{N}}\left((g \bar{n})^{-1} y_{i}^{*}\right)\right) \otimes P_{N}\left((g \bar{n})^{-1} y_{i}\right) .
$$

Now

$$
\begin{aligned}
& \sum_{i}\left(f(g \bar{n}) \circ P_{\bar{N}}\left((g \bar{n})^{-1} y_{i}^{*}\right)\right) \otimes P_{N}\left((g \bar{n})^{-1} y_{i}\right) \\
& \quad=\sum_{i}\left(f(g \bar{n}) \circ P_{\bar{N}}\left(y_{i}^{*}\right)\right) \otimes P_{N}\left(y_{i}\right)=f(g \bar{n})
\end{aligned}
$$

by Remark (1.1.6), since $f(g \bar{n}) \in \sigma_{\mu+\mu_{1}}=P_{\mu+\mu_{1}}^{M}\left(\sigma_{\mu} \otimes \sigma_{\mu_{1}}\right) \subseteq \sigma_{\mu} \otimes \sigma_{\mu_{1}}$, and the underlying space of $\sigma_{\mu_{1}}$ is $F^{N}$.

Therefore,

$$
\begin{aligned}
S(J(\bar{P}: P, \mu, \nu) \otimes I) T f(g) & =P_{\mu+\mu_{1}}^{M}\left(\int_{\bar{N}} f(g \bar{n}) d \bar{n}\right)=\int_{\bar{N}} f(g \bar{n}) d \bar{n} \\
& =J\left(\bar{P}: P, \mu+\mu_{1}, \nu+\nu_{1}\right) f(g),
\end{aligned}
$$

which is the desired commutativity.

2.5. Vogan's Lemma. The following lemma is due to Vogan [V1].

(2.5.1) Lemma. Let $\mathfrak{g}$ be a complex reductive Lie algebra, $I \subseteq \mathscr{U}(\mathfrak{g})$ any two-sided ideal, and let $\mathscr{T}_{I}$ denote the category of $\mathscr{U}(\mathfrak{g})$-modules $M$ such that $I \cdot M \equiv 0$. Let $\phi$ be any natural construction which associates to each object $X$ in $\mathscr{T}_{I}$ the following $\mathscr{U}(\mathfrak{g})$-module map:

$$
\phi_{X}: X \rightarrow X .
$$


Then there is a unique element $\bar{Z} \in \mathscr{Z}(g) / I \cap \mathscr{Z}(g)$ such that, for all $X \in \mathscr{T}_{I}$,

$$
\phi_{X}(x)=Z \cdot x \quad(x \in X) .
$$

Here $Z$ is a representative of $\bar{Z}$ in $\mathscr{Z}(\mathfrak{g})$. In particular, if $I \cap \mathscr{Z}(\mathfrak{g})$ is maximal in $\mathscr{Z}(\mathfrak{g})$, then there is a constant $c \in \mathbb{C}$ such that

$$
\phi_{X}(x)=c x, \quad x \in X .
$$

(2.5.2) Remark. A formula for $Z$ is also given in [V1], namely, $Z=\phi_{N_{l}}(\pi(1))$, where $N_{I}=\mathscr{U}(g) / I$ and $\pi$ is the natural map from $\mathscr{U}(\mathfrak{g})$ to $N_{I}$.

From the above lemma, it follows

(2.5.3) Proposition. Let $G$ be a real reductive Lie group, $\mathscr{T}_{\lambda}$ the category of $\mathscr{U}\left(\mathfrak{g}_{\mathbb{C}}\right)$-modules having the infinitesimal character $\lambda \in \mathscr{C}_{G}\left(\mathscr{C}_{G}\right.$ is a fixed positive Weyl chamber as in $\S 2.1)$, and $F_{\Lambda}$ the finite-dimensional irreducible representation of $G$ with the highest weight $\Lambda$. Let $X \in \mathscr{T}_{\lambda}$ and define $\phi: X \rightarrow X$ by

$$
\phi(x)=\sum_{i} P_{\lambda+\Lambda}^{G}\left(x \otimes y_{i}\right) \circ y_{i}^{*},
$$

where $\left\{y_{i}\right\}$ and $\left\{y_{i}^{*}\right\}$ are any dual bases of $F_{\Lambda}$ and $F_{\Lambda}^{*}$, and $\circ$ is the contraction between $F_{\Lambda}$ and $F_{\Lambda}^{*}$. Then there exists a scalar $r_{\lambda}^{G}(\Lambda)$ depending on $\lambda$ and $\Lambda$ such that $\phi(x)=r_{\lambda}^{G}(\Lambda) x \forall x \in X$.

(2.5.4) Remark. Similarly, define

$$
\psi(x)=\sum_{i} P_{\lambda}^{G}\left(x \otimes v_{i}^{*}\right) \circ v_{i}, \quad x \in X \in \mathscr{T}_{\lambda+\Lambda} .
$$

Then there exists a scalar $R_{\lambda+\Lambda}^{G}(-\Lambda)$ depending on $\lambda$ and $\Lambda$ such that $\psi(x)=$ $R_{\lambda+\Lambda}^{G}(-\Lambda) x \forall x \in X$.

2.6. Properties of $T, S, U, V$. We refer the reader to $\S 2.3$ for the definitions of $T, S, U, V$. We first give a simple lemma about the induction functor.

(2.6.1) Lemma. $\operatorname{Ind}_{M A N}^{G}$ and $\operatorname{Ind}_{M A \bar{N}}^{G}$ are exact functors.

Proof. As an induction functor, it is always left exact. Since $G=K M A N$, restricting $\operatorname{Ind}_{M A N}^{G}$ to $K$ gives an isomorphism.

Since

$$
\operatorname{Ind}_{M A N}^{G} \mid K \cong \operatorname{Ind}_{M \cap K}^{K},
$$

and $\operatorname{Ind}_{M \cap K}^{K}$ is right exact by the "unitary trick," we see that $\operatorname{Ind}_{M A N}^{G}$ is also right exact.

We observe that

(i) $T_{0}$ is injective.

(ii) $S_{0}$ is surjective.

The exactness of the two induction functors implies

(2.6.2) Proposition. $T$ is injective and $S$ is surjective.

Assume $r_{\mu}^{M}\left(\mu_{1}\right) \neq 0$, and let $\left\{w_{j}\right\}$ and $\left\{w_{j}^{*}\right\}$ be any dual bases of $\sigma_{\mu_{1}}$ and $\sigma_{\mu_{1}}^{*}$. Thus, by Proposition (2.5.3), we have

$$
\frac{1}{r_{\mu}^{M}\left(\mu_{1}\right)} \sum_{j} P_{\mu+\mu_{1}}^{M}\left(u \otimes w_{j}\right) \circ w_{j}^{*}=u, \quad u \in \sigma_{\mu} .
$$


The above clearly implies

(iii) $\operatorname{span}_{v^{*} \in \sigma_{\mu_{1}}^{*}}\left\{T_{0}\left(\sigma_{\mu+\mu_{1}}\right) \circ v^{*}\right\}=\operatorname{span}_{v^{*} \in \sigma_{\mu_{1}}^{*}}\left\{\left(\sigma_{\mu+\mu_{1}}\right) \circ v^{*}\right\}=\sigma_{\mu}$.

(iv) If $u \in \sigma_{\mu}$ has the property that $S_{0}\left(u \otimes u_{1}\right)=P_{\mu+\mu_{1}}^{M}\left(u \otimes u_{1}\right)=0 \forall u_{1} \in \sigma_{\mu_{1}}$, then $u=0$.

Again the exactness of the two induction functors implies

$$
\operatorname{span}_{v^{*} \in F_{\mu_{1}, \nu_{1}}^{*}}\left\{T\left(I_{P, \mu+\mu_{1}, \nu+\nu_{1}}\right) \circ v^{*}\right\}=I_{P, \mu, \nu},
$$

and if $S(h \otimes v)=0 \quad \forall v \in F$, then $h=0$.

Since

$$
\begin{aligned}
& U\left(f \otimes v^{*}\right)=T(f) \circ v^{*} \quad(\text { by }(2.3 .7)), \\
& V(h)=\sum S\left(h \otimes v_{i}\right) \otimes v_{i}^{*} \quad(\text { by }(2.3 .13)),
\end{aligned}
$$

we immediately have

(2.6.4) Proposition. Assume $r_{\mu}^{M}\left(\mu_{1}\right) \neq 0$. Then $U$ is surjective and $V$ is injective.

The proofs of the following two propositions can be copied almost word for word from [VW]. We give some details for the sake of completeness.

(2.6.5) Proposition. There exists a nonzero complex valued polynomial $\phi_{1}$ on $\mathfrak{a}_{\mathbb{C}}^{*}$ such that, if $\phi_{1}(\nu) \neq 0$, then

$$
T: I_{P, \mu+\mu_{1}, \nu+\nu_{1}} \rightarrow P_{\mu+\mu_{1}, \nu+\nu_{1}}^{G}\left(I_{P, \mu, \nu} \otimes F_{\mu_{1}, \nu_{1}}\right)
$$

and

$$
S: P_{\mu+\mu_{1}, \nu+\nu_{1}}^{G}\left(I_{\bar{P}, \mu, \nu} \otimes F_{\mu_{1}, \nu_{1}}\right) \rightarrow I_{\bar{P}, \mu+\mu_{1}, \nu+\nu_{1}}
$$

are linear bijections.

Proof. Let $F=F_{1} \supset F_{2} \supset \cdots \supset F_{r} \supset F_{r+1}=(0)$ be a Jordan-Holder series for $F=F_{\mu_{1}, \nu_{1}}$ as a $P$-module. We may assume that $F_{r}$ is $\sigma_{\mu_{1}} \otimes a^{\nu} \otimes 1$ as a $P=M A N$-module. If $V$ is an $(\mathfrak{m}, K \cap M)$-module and if $\lambda \in \mathfrak{a}_{\mathbb{C}}^{*}$, then we denote by $V_{\lambda}$ the $(\mathfrak{p}, K \cap M)$-module $V$ with $\mathfrak{n}$ acting trivially, $\mathfrak{m}$ acting as it did on $V$, and $\mathfrak{a}$ acting by $\lambda$. Then each $F_{i} / F_{i+1}$ is of the form $\left(V_{i}\right)_{\lambda_{i}}$ with $V_{i}$ an irreducible finite-dimensional $(\mathfrak{m}, K \cap M)$-module. Thus $I_{P, \mu, \nu} \otimes F$ has a composition series $I_{P, \mu, \nu} \otimes F=M_{1} \supset M_{2} \supset \cdots \supset M_{r} \supset M_{r+1}=(0)$ with

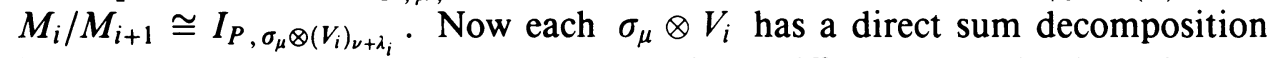
into ( $\mathrm{m}, K \cap M)$-submodules $V_{i j}$ each having a different (meaning inequivalent under the Weyl group) infinitesimal character (see $\S 2.1$ ). Thus, $I_{P, \sigma, \nu} \otimes F$ has a composition series with intermediate quotients $I_{P}, V_{i j}, \nu+\lambda_{i}$. Here $\lambda_{i}$ is a weight of the action of $\mathfrak{a}$ on $F$ and if $\lambda_{i}=\nu_{1}$, then $i=r$, and there is only one $j$ with $V_{r j} \cong \sigma_{\mu+\mu_{1}}$ (cf. the definition of $\sigma_{\mu+\mu_{1}}$ in $\S 2.3$ ). Let $\mu_{i j}$ be the infinitesimal character parameter for $V_{i j}$. Then

$$
\begin{aligned}
& \chi_{\left(\mu_{i j}, \nu+\lambda_{i}\right)}(C)-\chi_{\left(\mu+\mu_{1}, \nu+\nu_{1}\right)}(C) \\
&=\left(\left(\mu_{i j}, \nu+\lambda_{i}\right),\left(\mu_{i j}, \nu+\lambda_{i}\right)\right) \\
&-\left(\left(\mu+\mu_{1}, \nu+\nu_{1}\right),\left(\mu+\mu_{1}, \nu+\nu_{1}\right)\right) \\
&=\left(\mu_{i j}, \mu_{i j}\right)-\left(\mu+\mu_{1}, \mu+\mu_{1}\right)+\left(\lambda_{i}, \lambda_{i}\right) \\
&-\left(\nu_{1}, \nu_{1}\right)+2\left(\nu, \lambda_{i}-\nu_{1}\right)=\phi_{i j}(\nu),
\end{aligned}
$$


where $C$ is the second order Casimir operator of $G$. It is obvious that $\phi_{i j}(\nu)$ is a nonzero polynomial of $\nu$ for $i<r$.

Set $\phi_{1}=\prod_{\{i<r, j\}} \phi_{i j}$. Then if $\phi_{1}(\nu) \neq 0$, the only $I_{P, V_{i j}, \nu+\lambda_{i}}$ which can have the infinitesimal character $\left(\mu+\mu_{1}, \nu+\nu_{1}\right)$ is the one with $i=r$ and $V_{r j} \cong$ $\sigma_{\mu+\mu_{1}}$, and so $P_{\mu+\mu_{1}, \nu+\nu_{1}}^{G}\left(I_{P, \mu, \nu} \otimes F\right) \cong I_{P, \mu+\mu_{1}, \nu+\nu_{1}}$. Similarly, if $\phi_{1}(\nu) \neq 0$, then $P_{\mu+\mu_{1}, \nu+\nu_{1}}^{G}\left(I_{\bar{P}, \sigma, \nu} \otimes F\right) \cong I_{\bar{P}}, \mu+\mu_{1}, \nu+\nu_{1}$. Since $T$ is injective and $S$ is surjective, the proposition now follows.

In view of Proposition (2.6.4), a similar argument as in above gives the following

(2.6.6) Proposition. Assume $r_{\mu}^{M}\left(\mu_{1}\right) \neq 0$. Then there exists a nonzero complex valued polynomial $\phi_{2}$ on $\mathfrak{a}_{\mathbb{C}}^{*}$ such that, if $\phi_{2}(\nu) \neq 0$, then

$$
U: P_{\mu, \nu}^{G}\left(I_{P, \mu+\mu_{1}, \nu+\nu_{1}} \otimes F_{\mu_{1}, \nu_{1}}^{*}\right) \rightarrow I_{P, \mu, \nu}
$$

and

are linear bijections.

$$
V: I_{\bar{P}, \mu, \nu} \rightarrow P_{\mu, \nu}^{G}\left(I_{\bar{P}, \mu+\mu_{1}, \nu+\nu_{1}} \otimes F_{\mu_{1}, \nu_{1}}^{*}\right)
$$

(2.6.7) Remark. $r_{\mu}^{M}\left(\mu_{1}\right) \neq 0$ if $\mu$ is a dominant integral regular infinitesimal character (see $\S 2.8)$.

2.7. Second commuting diagram: functional equations for the intertwining operators. Again choose a constant $c$ such that, if $\operatorname{Re}(\nu, \alpha) \geqslant c$ for $\alpha \in \Phi(P, A)$, then both integrals defining $J(\bar{P}: P, \mu, \nu)$ and $J\left(\bar{P}: P, \mu+\mu_{1}, \nu+\nu_{1}\right)$ converge absolutely.

Assume $r_{\mu}^{M}\left(\mu_{1}\right) \neq 0$. Our main result is the following

(2.7.1) Theorem. If $\phi_{1}(\nu) \neq 0, \phi_{2}(\nu) \neq 0$ (see $\S 2.6$ for their definitions), $\operatorname{Re}(\nu, \alpha) \geqslant c$ for $\alpha \in \Phi(P, A)$, then the following diagram is commutative.

$$
\begin{array}{ccc}
P_{\mu, \nu}^{G}\left(I_{P, \mu+\mu_{1}, \nu+\nu_{1}} \otimes F_{\mu_{1}, \nu_{1}}^{*}\right) & \stackrel{r_{\mu, \nu}^{G}\left(\mu_{1}, \nu_{1}\right) J\left(\mu+\mu_{1}, \nu+\nu_{1}\right) \otimes I}{\longrightarrow} & P_{\mu, \nu}^{G}\left(I_{\bar{P}, \mu+\mu_{1}, \nu+\nu_{1}} \otimes F_{\mu_{1}, \nu_{1}}^{*}\right) \\
I_{P, \mu, \nu} & V \uparrow \\
& J(\mu, \nu) & I_{\bar{P}, \mu, \nu}
\end{array}
$$

Proof. Let $\left\{y_{i}\right\}$ and $\left\{y_{i}^{*}\right\}$ be dual bases of $F_{\mu_{1}, \nu_{1}}$ and $F_{\mu_{1}, \nu_{1}}^{*}$, as before, and let $f=\sum_{i} f_{i} \otimes y_{i}^{*}$ be any element in $P_{\mu, \nu}^{G}\left(I_{P, \mu+\mu_{1}, \nu+\nu_{1}} \otimes F_{\mu_{1}, \nu_{1}}^{*}\right)$. Then

$$
\left(J\left(\mu+\mu_{1}, \nu+\nu_{1}\right) \otimes I\right)\left(\sum_{i} f_{i} \otimes y_{i}^{*}\right) \in P_{\mu, \nu}^{G}\left(I_{\bar{P}, \mu+\mu_{1}, \nu+\nu_{1}} \otimes F_{\mu_{1}, \nu_{1}}^{*}\right) .
$$

Since $r_{\mu}^{M}\left(\mu_{1}\right) \neq 0$ and $\phi_{2}(\nu) \neq 0, V$ is a linear isomorphism by Proposition (2.6.6).

Therefore, there is a unique $h \in I_{\bar{P}, \mu, \nu}$ such that

$$
\begin{aligned}
(J(\mu & \left.\left.+\mu_{1}, \nu+\nu_{1}\right) \otimes I\right)\left(\sum_{i} f_{i} \otimes y_{i}^{*}\right) \\
& =\sum_{i} J\left(\mu+\mu_{1}, \nu+\nu_{1}\right) f_{i} \otimes y_{i}^{*}=V(h) .
\end{aligned}
$$


By (2.3.13),

$$
V(h)=\sum_{i} S\left(h \otimes y_{i}\right) \otimes y_{i}^{*}
$$

so

$$
\sum_{i} J\left(\mu+\mu_{1}, \nu+\nu_{1}\right) f_{i} \otimes y_{i}^{*}=\sum_{i} S\left(h \otimes y_{i}\right) \otimes y_{i}^{*},
$$

and hence

$$
J\left(\mu+\mu_{1}, \nu+\nu_{1}\right) f_{i}=S\left(h \otimes y_{i}\right), \quad \text { for each } i .
$$

By the first commuting diagram (2.4.2), namely,

$$
S(J(\mu, \nu) \otimes I) T=J\left(\mu+\mu_{1}, \nu+\nu_{1}\right),
$$

we obtain

$$
\begin{aligned}
S(J(\mu, \nu) \otimes I) T f_{i} & =J\left(\mu+\mu_{1}, \nu+\nu_{1}\right) f_{i}=S\left(h \otimes y_{i}\right) \\
& =S\left[P_{\mu+\mu_{1}, \nu+\nu_{1}}^{G}\left(h \otimes y_{i}\right)\right] .
\end{aligned}
$$

Since $\phi_{1}(\nu) \neq 0, S$ is a linear isomorphism by Proposition (2.6.5), and so

$$
(J(\mu, \nu) \otimes I) T f_{i}=P_{\mu+\mu_{1}, \nu+\nu_{1}}^{G}\left(h \otimes y_{i}\right) .
$$

Thus, by contracting with $y_{i}^{*}$ and summing over $i$, we obtain by Proposition (2.5.3)

$$
\sum_{i}\left((J(\mu, \nu) \otimes I) T f_{i}\right) \circ y_{i}^{*}=\sum_{i} P_{\mu+\mu_{1}, \nu+\nu_{1}}^{G}\left(h \otimes y_{i}\right) \circ y_{i}^{*}=r_{\mu, \nu}^{G}\left(\mu_{1}, \nu_{1}\right) h .
$$

The left-hand side is equal to

$$
\begin{aligned}
\sum_{i} J(\mu, \nu)\left(T f_{i} \circ y_{i}^{*}\right) & =\sum_{i} J(\mu, \nu) U\left(f_{i} \otimes y_{i}^{*}\right) \quad(\text { by }(2.3 .7)) \\
& =J(\mu, \nu) U\left(\sum_{i} f_{i} \otimes y_{i}^{*}\right)
\end{aligned}
$$

Thus, we obtain

$$
J(\mu, \nu) U\left(\sum_{i} f_{i} \otimes y_{i}^{*}\right)=r_{\mu, \nu}^{G}\left(\mu_{1}, \nu_{1}\right) h
$$

So,

$$
\begin{aligned}
r_{\mu, \nu}^{G}( & \left.\mu_{1}, \nu_{1}\right)\left(J\left(\mu+\mu_{1}, \nu+\nu_{1}\right) \otimes I\right)\left(\sum_{i} f_{i} \otimes y_{i}^{*}\right) \\
= & r_{\mu, \nu}^{G}\left(\mu_{1}, \nu_{1}\right) V(h) \quad(\text { by }(2.7 .3)) \\
& =V J(\mu, \nu) U\left(\sum_{i} f_{i} \otimes y_{i}^{*}\right) \quad(\text { by }(2.7 .4)),
\end{aligned}
$$

which is exactly the commutativity of the diagram (2.7.2). 
(2.7.5) Remark. In [VW], a similar commutative diagram is proved with $F$ an irreducible finite-dimensional spherical representation. Our proof is different from theirs.

(2.7.6) Remark. Since each representation present in diagram (2.7.2) is generically irreducible, one knows a priori that this diagram is commutative up to a multiple generically (Schur's Lemma).

To get this multiple, one forms $\sum_{i} V J(\mu, \nu) U P_{\mu, \nu}^{G}\left(f \otimes y_{i}^{*}\right) \circ y_{i}$ and computes as follows:

$$
\begin{array}{rl}
\sum_{i} V & J(\mu, \nu) U P_{\mu, \nu}^{G}\left(f \otimes y_{i}^{*}\right) \circ y_{i} \\
& =\sum_{i} V J(\mu, \nu) U\left(f \otimes y_{i}^{*}\right) \circ y_{i} \\
& =\sum_{i, j} S\left(J(\mu, \nu) U\left(f \otimes y_{i}^{*}\right) \otimes y_{j}\right) \otimes y_{j}^{*} \circ y_{i} \quad(\text { by }(2.3 .13)) \\
& =\sum_{i} S\left(J(\mu, \nu) U\left(f \otimes y_{i}^{*}\right) \otimes y_{i}\right) \\
& =\sum_{i} S(J(\mu, \nu) \otimes I)\left(U\left(f \otimes y_{i}^{*}\right) \otimes y_{i}\right) \\
& =\sum_{i} S(J(\mu, \nu) \otimes I) T f \quad\left(\text { since } U=T^{\dagger},(\text { see } 2.3 .7)\right) \\
& =J\left(\mu+\mu_{1}, \nu+\nu_{1}\right) f \quad(\text { by the commutativity of diagram (2.4.2)). }
\end{array}
$$

Thus,

$$
\begin{aligned}
& R_{\mu+\mu_{1}, \nu+\nu_{1}}^{G}\left(-\mu_{1},-\nu_{1}\right) \sum_{i} V J(\mu, \nu) U P_{\mu, \nu}^{G}\left(f \otimes y_{i}^{*}\right) \circ y_{i} \\
& \quad=R_{\mu+\mu_{1}, \nu+\nu_{1}}^{G}\left(-\mu_{1},-\nu_{1}\right) J\left(\mu+\mu_{1}, \nu+\nu_{1}\right) f \\
& \quad=\sum_{i} P_{\mu, \nu}^{G}\left(J\left(\mu+\mu_{1}, \nu+\nu_{1}\right) f \otimes y_{i}^{*}\right) \circ y_{i} \quad \text { (by Remark (2.5.4)). }
\end{aligned}
$$
have

Combining the above identity with the commutativity of diagram (2.7.2), we (2.7.7) Corollary. If $r_{\mu}^{M}\left(\mu_{1}\right) \neq 0, \phi_{1}(\nu) \neq 0, \phi_{2}(\nu) \neq 0$, and $\operatorname{Re}(\nu, \alpha) \geqslant c$ for $\alpha \in \Phi(P, A)$, then

$$
r_{\mu, \nu}^{G}\left(\mu_{1}, \nu_{1}\right) R_{\mu+\mu_{1}, \nu+\nu_{1}}^{G}\left(-\mu_{1},-\nu_{1}\right)=1 .
$$

2.8. About the factor $r_{\lambda}^{G}(\Lambda)$. Recall the definition of $r_{\lambda}^{G}(\Lambda)$ in $\S 2.5$.

(2.8.1) Proposition (Wallach). If $\lambda$ is a dominant integral regular infinitesimal character, then

$$
r_{\lambda}(\Lambda)=\frac{\prod_{\alpha>0}(\lambda+\Lambda, \alpha)}{\prod_{\alpha>0}(\lambda, \alpha)}
$$

Proof. Let $\rho_{G}$ be the half sum of positive $\mathfrak{h}$ roots in $\mathfrak{g}_{\mathbb{C}}$, as usual. Let $F_{\lambda-\rho_{G}}$ be the irreducible finite-dimensional $\mathfrak{g}_{\mathbb{C}}$-module with highest weight $\lambda-\rho_{G}$, which exists by our assumption on $\lambda$. By Proposition (2.5.3), we have

$$
\sum_{i} P_{\lambda+\Lambda}^{G}\left(m \otimes y_{i}\right) \circ y_{i}^{*}=r_{\lambda}^{G}(\Lambda) m, \quad m \in F_{\lambda-\rho_{G}} .
$$


Let $\left\{w_{j}\right\}$ and $\left\{w_{j}^{*}\right\}$ be dual bases of $F_{\lambda-\rho_{G}}$ and $F_{\lambda-\rho_{G}}^{*}$. In particular, the above identity gives

$$
\sum_{i} P_{\lambda+\Lambda}^{G}\left(w_{j} \otimes y_{i}\right) \circ y_{i}^{*}=r_{\lambda}^{G}(\Lambda) w_{j} \quad \forall j .
$$

Hence by contracting with $w_{j}^{*}$ and summing over $j$, we obtain

$$
\sum_{j} \sum_{i} P_{\lambda+\Lambda}^{G}\left(w_{j} \otimes y_{i}\right) \circ y_{i}^{*} \circ w_{j}^{*}=r_{\lambda}^{G}(\Lambda) \sum_{j} w_{j} \circ w_{j}^{*}=r_{\lambda}^{G}(\Lambda) \operatorname{dim} F_{\lambda-\rho_{G}} .
$$

Since the left-hand side is independent of the choice of dual bases $\left\{w_{j} \otimes y_{i}\right\}$ and $\left\{w_{j}^{*} \otimes y_{i}^{*}\right\}$ of $F_{\lambda-\rho_{G}} \otimes F_{\Lambda}$ and $\left(F_{\lambda-\rho_{G}} \otimes F_{\Lambda}\right)^{*}$, we have

$$
\text { (2.8.2) }=\text { trace of } P_{\lambda+\Lambda}^{G}=\operatorname{dim} P_{\lambda+\Lambda}^{G}\left(F_{\lambda-\rho_{G}} \otimes F_{\Lambda}\right)=\operatorname{dim} F_{\lambda+\Lambda-\rho_{G}} .
$$

Thus,

$$
r_{\lambda}^{G}(\Lambda)=\frac{\operatorname{dim} F_{\lambda+\Lambda-\rho_{G}}}{\operatorname{dim} F_{\lambda-\rho_{G}}}=\frac{\prod_{\alpha>0}(\lambda+\Lambda, \alpha) / \prod_{\alpha>0}\left(\rho_{G}, \alpha\right)}{\prod_{\alpha>0}(\lambda, \alpha) \prod_{\alpha>0}\left(\rho_{G}, \alpha\right)}=\frac{\prod_{\alpha>0}(\lambda+\Lambda, \alpha)}{\prod_{\alpha>0}(\lambda, \alpha)}
$$

by the famous Weyl dimension formula.

Let $\mathscr{C}(\Lambda)$ be the region of $\lambda$ such that if $\tau$ is a h-weight of $F_{\Lambda}$ and $\tau \neq$ $\Lambda$, then $\chi_{\lambda+\tau}(C) \neq \chi_{\lambda+\Lambda}(C)$. Recall here that $C$ is the second order Casimir operator and $\chi_{\lambda+\tau}$ is the infinitesimal character associated to $\lambda+\tau \in \mathfrak{h}^{*}$ via the Harish-Chandra isomorphism (see $\S 2.1)$. Thus $\mathscr{C}(\Lambda)$ is the complement in $\mathfrak{h}^{*}$ of a finite number of hyperplanes.

The proof of the following proposition is essentially given in [VW].

(2.8.3) Proposition. $r_{\lambda}(\Lambda)$ is a rational function in $\mathscr{C}(\Lambda)$.

Proof. Let $N_{\lambda}=\mathscr{U}(\mathfrak{g}) / I_{\lambda}$, where $I_{\lambda}$ is the two-sided ideal of $\mathscr{U}\left(g_{\mathbb{C}}\right)$ generated by $z-\chi_{\lambda}(z), \quad z \in \mathscr{Z}\left(\mathfrak{g}_{\mathbb{C}}\right)$. Then

$$
N_{\lambda} \otimes F_{\Lambda}=\bigoplus_{\tau \in \pi\left(F_{\Lambda}\right)} P_{\lambda+\tau}^{G}\left(N_{\lambda} \otimes F_{\Lambda}\right),
$$

where $\pi\left(F_{\Lambda}\right)$ is the set of $\mathfrak{h}$-weights of $F_{\Lambda}$ (see [Ko]).

Let $r(\tau)$ be such that

$$
\left(C-\chi_{\lambda+\tau}(C)\right)^{r(\tau)} P_{\lambda+\tau}^{G}\left(N_{\lambda} \otimes F_{\Lambda}\right)=0 .
$$

Set

$$
U_{\lambda}=\prod_{\tau \in \pi(F)-\{\Lambda\}}\left(C-\chi_{\lambda+\tau}(C)\right)^{r(\tau)},
$$

$\tilde{Z}_{\lambda}=\chi_{\lambda+\Lambda}\left(U_{\lambda}\right)^{-1} U_{\lambda}$ (it makes sense since $\chi_{\lambda+\tau}(C) \neq \chi_{\lambda+\Lambda}(C)$, for $\tau \neq \Lambda$ ).

Then the projection of $N_{\lambda} \otimes F_{\Lambda}$ onto $P_{\lambda+\Lambda}^{G}\left(N_{\lambda} \otimes F_{\Lambda}\right)$ is given by the action of $\tilde{Z}_{\lambda}$ on $N_{\lambda} \otimes F_{\Lambda}$.

Let

$$
Z_{\lambda}=\sum \tilde{Z}_{\lambda}\left(1 \otimes y_{i}\right) \circ y_{i}^{*}
$$

Then $r_{\lambda}(\Lambda)=\chi_{\lambda}\left(Z_{\lambda}\right)$ by Remark (2.5.2). It is clear that $r_{\lambda}(\Lambda)$ is rational in $\lambda$.

Let $\Phi(\mathfrak{b}, \mathfrak{h})$ be the set of positive $\mathfrak{h}$ roots in $\mathfrak{g}_{\mathbb{C}}$ specified by our choice of Borel subalgebra $\mathfrak{b}$. The above proposition clearly implies 
(2.8.4) Corollary. There exists $a c>0$ such that $r_{\lambda}(\Lambda)$ is a rational function of $\lambda$ in the region $\{\lambda \mid \operatorname{Re}(\lambda, \alpha) \geqslant c$ for $\alpha \in \Phi(\mathfrak{b}, \mathfrak{h})\}$.

Combining Corollary (2.8.4) with Proposition (2.8.1), and observing that a nonzero rational function cannot have "half a lattice" as its zeros, we obtain

\section{(2.8.5) Proposition.}

$$
r_{\lambda}(\Lambda)=\frac{\prod_{\alpha>0}(\lambda+\Lambda, \alpha)}{\prod_{\alpha>0}(\lambda, \alpha)}
$$

if $\operatorname{Re}(\lambda, \alpha) \geqslant c$ for $\alpha \in \Phi(\mathfrak{b}, \mathfrak{h})$.

\section{REFERENCES}

[He] S. Helgason, Groups and geometric analysis, Academic Press, Orlando, Fla., 1984.

[Hu] J. Humphreys, Introduction to Lie algebras and representation theory, Graduate Texts in Math., vol. 9, Springer-Verlag, New York, 1987.

[Kn] A. Knapp, Representation theory of semisimple Lie groups: An overview based on examples, Princeton Univ. Press, Princeton, N.J., 1986.

[Ko] B. Kostant, On the tensor product of a finite and an infinite dimensional representation, $\mathrm{J}$. Funct. Anal. 20 (1975), 257-285.

[KS1] A. Knapp and E. Stein, Intertwining operators for semisimple groups, Ann. of Math. (2) 93 (1971), 489-578.

[KS2] _ Intertwining operators for semisimple groups. II, Invent. Math. 60 (1980), 9-84.

[Sl] S. Lang, $S l_{2}(\mathbb{R})$, Graduate Texts in Math., vol. 105, Springer-Verlag, 1975.

[V1] D. Vogan, Jr., Representations of real reductive Lie groups, Birkhäuser, Boston, Mass., 1981.

[VW] D. Vogan, Jr. and N. Wallach, Intertwining operators for real reductive groups, Adv. Math. 82 (1990), 203-243.

[W1] N. Wallach, Harmonic analysis on homogeneous spaces, Marcel Dekker, New York, 1973.

[Zh] C. Zhu, Two topics in harmonic analysis on reductive groups, Thesis, Yale University, 1990.

[Zu] G. Zuckerman, Tensor products of finite and infinite dimensional representations of semisimple Lie groups, Ann. of Math. (2) 106 (1977), 295-308. 20742

Department of Mathematics, University of Maryland, College Park, Maryland

Current address: Department of Mathematics, National University of Singapore, Kent Ridge, Singapore 0511

E-mail address: matzhucb@nuscc.nus.sg 